\title{
EQUIVARIANT STABLE HOMOTOPY AND FRAMED BORDISM
}

\author{
BY
}

\section{CZES KOSNIOWSKI}

ABSTRACT. This paper gives an elementary proof of the result that equivariant stable homotopy is the same as equivariant framed bordism.

1. Introduction. Let $G$ be a finite group. The purpose of this paper is to give an elementary proof of the result that equivariant stable homotopy and equivariant framed bordism, as $G$ homology theories, are the same.

The idea of the proof is as follows-full definitions and details will be found inside-let $\omega_{V}^{G}(X, A)$ denote equivariant framed bordism and let $\pi_{V}^{s, G}(X, A)$ denote equivariant stable homotopy. Then, for any subgroup $H$ of $G$, we have the following commutative diagram

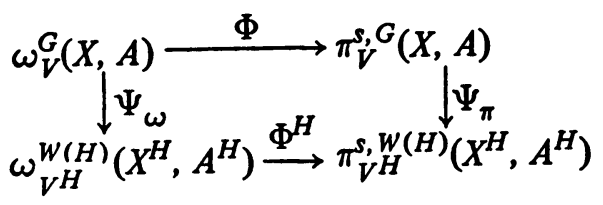

where $W(H)$ denotes the quotient $N(H) / H$ and $N(H)$ is the normaliser of $H$ in $G$. The maps $\Phi$ and $\Phi^{H}$ are the Pontrjagin-Thom maps, while $\Psi_{\omega}$ and $\Psi_{\pi}$ denote taking fixed point sets with respect to $H$.

To each subgroup $H$ of $G$, we can associate a pair of $G$ spaces $\left(E F, E F^{\prime}\right)$. If, in the commutative diagram, we replace $(X, A)$ by $(X, A) \times\left(E F, E F^{\prime}\right)=$ $\left(X \times E F, X \times E F^{\prime} \cup A \times E F\right)$ then we can show quite easily that

(1) $\Psi_{\omega}$ is an isomorphism,

(2) $\Phi^{H}$ is an isomorphism, and

(3) $\Psi_{\pi}$ is injective.

It therefore follows that $\Phi$ (and $\Psi_{\pi}$ ) is an isomorphism.

Next, to the group $G$ we can associate a finite sequence of $G$ spaces $\varnothing=$ $E F_{1} \subset E F_{2} \subset \cdots \subset E F_{n}$ with the following properties.

(1) For each $i, \Phi$ is an isomorphism for the space $(X, A) \times\left(E F_{i+1}, E F_{i}\right)$.

(2) For each $G$ homology theory there is a long exact sequence involving the spaces $(X, A) \times\left(E F_{j}, E F_{i}\right),(X, A) \times\left(E F_{k}, E F_{i}\right),(X, A) \times\left(E F_{k}, E F_{j}\right)$ for any $i<j<k$.

Received by the editors June 23, 1975.

AMS (MOS) subject classifications (1970). Primary 57D85, 55C35; Secondary 55B20, $55 \mathrm{E} 10$. 
(3) $E F_{n}$ is $G$ contractible.

So, by induction and the five lemma, the result easily follows.

This result, for $X$ a point, was first announced by G. Segal [12] with a proof to appear in J. J. O'Connor's thesis [9]. A proof by H. Hauschild was given in his thesis [6]. The result for $X$ a point also appears as a corollary in the thesis of R. Rubinsztein [11].

2. Equivariant framed bordism. Let $V$ be a $G$ module-i.e. a finite dimensional real vector space on which $G$ acts linearly, and let $M$ be a $G$ manifold.

If $\xi$ is a $G$ vector bundle on $M$, we say that $\xi$ has a $V$ trivialization if there exists some integer $n$ and a $G$ bundle isomorphism $\phi_{M}$ s.t.

$$
\phi_{M}: \xi \oplus\left(\mathrm{R}^{n} \times M\right) \cong(V \times M) \oplus\left(\mathrm{R}^{n} \times M\right)
$$

where $\mathbf{R}^{n}$ denotes the trivial $n$ dimensional $G$ module. A $V$ framed $G$ manifold is a $G$ manifold $M$ together with a $G$ homotopy equivalence class of $V$ trivializations of the tangent bundle of $M$.

Notice that this definition of a $V$ framed $G$ manifold differs from that used in [7] and [12] where $\mathrm{R}^{n}$ is replaced by any $G$ module $U$. However we do have the following result which will be needed later on.

LEMMA 2.1. If $M$ is a free $G$ manifold then the above two notions of $V$ framing are equivalent.

Proof. We need only show that if $T M \oplus(U \times M) \cong(V \times M) \oplus(U \times M)$ for some $G$ module $U$ then $T M \oplus\left(\mathrm{R}^{n} \times M\right) \cong(V \times M) \oplus\left(\mathrm{R}^{n} \times M\right)$ for some n. The $G$ vector bundles over $M$ are in a one-to-one correspondence with the vector bundles over $M / G$ (see [1]), the correspondence being given by $E \rightarrow E / G$, $\pi^{*}\left(E^{\prime}\right) \leftarrow E^{\prime}$ where $\pi: M \rightarrow M / G$. So

$$
T M / G \oplus(U \times M) / G \cong(V \times M) / G \oplus(U \times M) / G
$$

as vector bundles over $M / G$. Now, there exists some bundle $E$ over $M / G$ such that $(U \times M) / G \oplus E$ is a trivial vector bundle over $M / G$-say $\mathbf{R}^{n} \times M / G$. Thus we have

$$
T M / G \oplus\left(\mathrm{R}^{n} \times M\right) / G \cong(V \times M) / G \oplus\left(\mathrm{R}^{n} \times M\right) / G
$$

and

$$
T M \oplus\left(\mathrm{R}^{n} \times M\right) \cong(V \times M) \oplus\left(\mathrm{R}^{n} \times M\right) .
$$

Let $(X, A)$ be a $G$ topological pair; then a $V$ framed bordism element of $(X, A)$ is a pair $(M, f)$ where

(i) $M$ is a $V$ framed $G$ manifold, and 
(ii) $f: M \rightarrow X$ is an equivariant map with $f(\partial M) \subset A$. ( $\partial M$ denotes the boundary of $M$.)

If $M$ is a $V$ framed $G$ manifold, then we have a trivialization

$$
\phi_{M}: T M \oplus\left(\mathbf{R}^{n} \times M\right) \cong(V \times M) \oplus\left(\mathbf{R}^{n} \times M\right) .
$$

Let $-\phi_{M}$ denote the trivialization

$$
\begin{aligned}
-\phi_{M} & =\phi_{M} \oplus(-\mathrm{id}): T M \oplus\left(\mathbf{R}^{n} \times M\right) \oplus(\mathbf{R} \times M) \\
& \cong(V \times M) \oplus\left(\mathbf{R}^{n} \times M\right) \oplus(\mathbf{R} \times M)
\end{aligned}
$$

where $-\mathrm{id}: \mathbf{R} \times M \rightarrow \mathbf{R} \times M$ is the map defined by sending $(t, m)$ to $(-t, m)$. The manifold with this framing will be denoted by $-M$.

Two $V$ framed bordism elements $(M, f),\left(M, f^{\prime}\right)$ of $(X, A)$ are said to be equivalent if there exists a pair $(N, q)$ where

(i) $N$ is a $V \oplus \mathrm{R}$ framed $G$ manifold,

(ii) $M \cup\left(-M^{\prime}\right) \subset \partial N$, the induced $V$ framings on $\partial N$ restricted to $M$, $-M^{\prime}$ agreeing with that on $M,-M^{\prime}$ respectively,

(iii) $q: N \rightarrow X$ is an equivariant map with $q|M=f, q|\left(-M^{\prime}\right)=f^{\prime}$ and $q\left(\partial M\left(M \cup\left(-M^{\prime}\right)\right)\right) \subset A$.

The set of $V$ framed bordism elements of $(X, A)$ under this equivalence relationship forms an abelian group denoted by $\omega_{V}^{G}(X, A)$.

Let $V_{0}, V_{1}, \ldots, V_{r}$ be a complete set of irreducible nonisomorphic $\dot{G}$ modules-with $V_{0}$ being the trivial one dimensional $G$ module, i.e. R. Thus any $G$ module $V$ may be represented uniquely as a sum $V=\Sigma_{i=0}^{r} n_{i} V_{i}$ where the $n_{i}$ are integers $\left(n_{i} \geqslant 0\right)$ and $n_{i} V_{i}$ means the direct sum of $n_{i}$ copies of $V_{i}$.

An element $\alpha \in R O(G)$-the real representation ring of $G$-may be written as $\alpha=\Sigma_{i=0}^{r} \alpha_{i} V_{i}$ where the $\alpha_{i}$ are integers. Let $\alpha^{+}$be the sum $\Sigma \alpha_{j} V_{j}$ where $\alpha_{j}>0$ and let $\alpha^{-}$be the sum $\Sigma-\alpha_{k} V_{k}$ where $\alpha_{k}<0$. Then $\alpha=\alpha^{+}-\alpha^{-}$and each of $\alpha^{+}$and $\alpha^{-}$are $G$ modules. We define

$$
\omega_{\alpha}^{G}(X, A) \equiv \omega_{\alpha^{+}}^{G}\left(D\left(\alpha^{-}\right) \times X, S\left(\alpha^{-}\right) \times X \cup D\left(\alpha^{-}\right) \times A\right)
$$

where $D, S$ stand for the unit disc and unit sphere respectively.

The set $\left\{\omega_{\alpha}^{G}(X, A) ; \alpha \in R O(G)\right\}$ forms a $G$ homology theory indexed by elements $\alpha \in R O(G)$. (This theory has suspension isomorphisms for trivial $G$ modules $\mathbf{R}^{n}$, although later on it will follow that we have suspension isomorphisms for all $G$ modules.)

3. Equivariant stable homotopy. We recall [7] the definition of equivariant stable homotopy. If $V$ is a $G$ module, let $S^{V}$ denote its one point compactification, in which $\infty$ is regarded as base point. We say that a $G$ module $W$ is 
admissible if $W$ contains at least one copy of each irreducible $G$ module-for example, $W$ may be $\Sigma_{i=0}^{r} V_{i}$ where the $V_{i}$ are as defined in $\S 2$.

We define $\pi_{\alpha}^{s, G}(X, A)$ to be the direct limit (over $k \in \mathrm{Z}$ ) of the $G$ homotopy classes of base point preserving $G$ maps from $S^{k W \oplus \alpha^{+}}$to $S^{k W \oplus \alpha^{-} \wedge}$ $\left(X^{+} / A^{+}\right)$. In symbols

The maps

$$
\pi_{\alpha}^{s, G}(X, A)=\lim _{k \rightarrow \infty}\left[S^{k W \oplus \alpha^{+}} ; S^{k W \oplus \alpha^{-}} \wedge\left(X^{+} / A^{+}\right)\right]_{G}^{0}
$$

$$
\begin{aligned}
& {\left[S^{k W \oplus \alpha^{+}} ; S^{k W \oplus \alpha^{-}} \wedge\left(X^{+} / A^{+}\right)\right]_{G}^{0} } \\
& \rightarrow\left[S^{(k+1) W \oplus \alpha^{+}} ; S^{\left.(k+1) W \oplus \alpha^{-} \wedge\left(X^{+} / A^{+}\right)\right]_{G}^{0}}\right.
\end{aligned}
$$

are given by suspending with $S^{W}$.

This definition is independent of the choice of $W$-so long as $W$ contains at least one copy of each irreducible $G$ module-see [7].

The set $\left\{\pi_{\alpha}^{s, G}(X, A) ; \alpha \in R O(G)\right\}$ forms a $G$ homology theory and has suspension isomorphisms for all $G$ modules $V$, i.e.

$$
\pi_{\alpha}^{s, G}(X, A) \cong \pi_{\alpha \oplus V}^{s, G}(D(V) \times X, S(V) \times X \cup D(V) \times A),
$$

in other words it forms a $G$ homology theory in the sense of [7].

4. The commutative diagram. Since

$$
h_{\alpha}^{G}(X, A)=h_{\alpha^{+}}^{G}\left(D\left(\alpha^{-}\right) \times X, S\left(\alpha^{-}\right) \times X \cup D\left(\alpha^{-}\right) \times A\right),
$$

in the case that $h=\omega$ or $h=\pi$, we shall henceforth only look at $h_{V}^{G}(X, A)$ where $V$ is some $G$ module.

The aim of this section is to show the existence of the following commutative diagram.

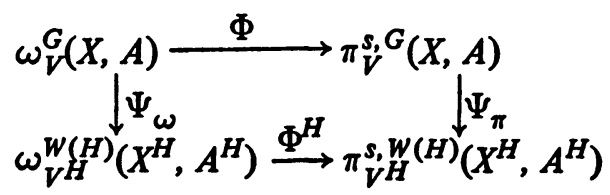

(a) The map $\Phi$ is given by the Pontrjagin-Thom construction, which we proceed to describe.

Let $(M, f)$ be a $V$ framed bordism element of $(X, A)$. We know that $T M$ $\oplus\left(\mathbf{R}^{n} \times M\right) \cong\left(V \oplus \mathbf{R}^{n}\right) \times M$, also we may embed $M$ in $(k-n) W$ where $k$ is some large number and $W$ is admissible in the sense of $\S 3$. If $\nu(M,(k-n) W)$ denotes the normal bundle of $M$ in $(k-n) W$ then we have the following bundle isomorphisms: 
$T M \oplus \nu(M,(k-n) W) \cong(k-n) W \times M$,

$(V \times M) \oplus\left(\mathrm{R}^{n} \times M\right) \oplus \nu(M,(k-n) W) \cong\left(\mathrm{R}^{n} \times M\right) \oplus((k-n) W \times M)$

so

$$
\nu\left(M,(k-n) W \oplus \mathrm{R}^{n} \oplus V\right) \cong\left(\mathrm{R}^{n} \times M\right) \oplus((k-n) W \times M)
$$

and thus

$$
\nu(M, k W \oplus V) \cong k W \times M .
$$

In other words we can embed $M$ in $k W \oplus V$ for some large $k$, such that the normal bundle is $k W \times M$. Consider the following sequence of maps:

$$
\begin{aligned}
s^{k W \oplus V} & =D(k W \oplus V) / S(k W \oplus V) \rightarrow D(\nu) /(D(\nu \mid \partial M) \cup S(\nu)) \\
& \cong(M \times D(k W)) /((\partial M \times D(k W)) \cup(M \times S(k W))) \\
& \stackrel{f \times \mathrm{id}}{\longrightarrow}(X \times D(k W)) /((A \times D(k W)) \cup(X \times S(k W))) .
\end{aligned}
$$

The composite defines an element of $\pi_{V}^{s, G}(X, A)$ and so defines the map $\Phi$.

(b) If $(M, f)$ is a $V$ framed bordism element of $(X, A)$ then $M^{H}$ is a $V^{H}$ framed $W(H)$ manifold and $f \mid M^{H}: M^{H} \rightarrow X^{H}$ is a $W(H)$ equivariant map with $f\left(\partial M^{H}\right) \subset A^{H}$. We therefore define $\psi_{\omega}(M, f)$ to be $\left(M^{H}, f \mid M^{H}\right)$.

(c) The map $\Phi^{H}$ is the Pontrjagin-Thom construction as in (a).

(d) $\Psi_{\pi}$ is defined by taking fixed point sets with respect to $H$, i.e.

$$
\left[S^{k W \oplus V} ; S^{k W} \wedge X / A\right]_{G}^{0} \rightarrow\left[S k W^{H} \oplus V^{H} ; S^{k} W^{H} \wedge X^{H} / A^{H}\right]_{W(H)}^{0}
$$

( $W^{H}$ is clearly admissible for the group $W(H)$.)

The diagram is clearly commutative.

5. Families. Recall that a family $F$ in $G$ is a collection of subgroups of $G$ such that

(a) if $H \in F$ and $K \subset H$, then $K \in F$ and

(b) if $H \in F$ and $g \in G$, then $g \mathrm{Hg}^{-1} \in F$.

Following Palais [10], see also Bredon [2], we define universal spaces $E F$ as follows. If $H$ is a subgroup of $G$, let $E W(H)$ denote the universal $W(H)$ space (i.e. a contractible free $W(H)$ space such that $E W(H) \rightarrow E W(H) / W(H)=B W(H)$ is a numerable $W(H)$ principal bundle). Let $E F$ be defined by

$$
E F=*\left(G \times{ }_{N(H)} E W(H)\right)
$$

where the join is taken over a complete set of conjugacy classes of subgroups $H$ 
in F. (Note. The join used here is not the Palais join as in [10] and [2] -this is needed only if $F$ is not a family.)

We have, in particular, if $X$ is a $G$ space all of whose isotropy subgroups belong to $F$ then there is a unique map (up to $G$ homotopy) $X \rightarrow E F$.

Given a $G$ homology theory $h^{G}$ we define a new $G$ homology theory $h^{G}\left[F, F^{\prime}\right]$ for pairs $F^{\prime} \subset F$ of families in $G$ by

$$
h_{*}^{G}\left[F, F^{\prime}\right](X, A)=h_{*}^{G}\left(X \times E F, A \times E F \cup X \times E F^{\prime}\right) .
$$

This idea, in this form, comes from tom Dieck [5]. That $h^{G}\left[F, F^{\prime}\right]$ is indeed a $G$ homology theory is not too difficult to prove. For example, the long exact sequence

$(5.1) \cdots \rightarrow h_{*}^{G}\left[F, F^{\prime}\right](A) \rightarrow h_{*}^{G}\left[F, F^{\prime}\right](X) \rightarrow h_{*}^{G}\left[F, F^{\prime}\right](X, A) \rightarrow \cdots$

is obtained by looking at the associated $h^{G}$ theory long exact sequence of the triple $\left(X \times E F, X \times E F^{\prime} \cup A \times E F, X \times E F^{\prime}\right)$ and using the fact that

$$
\begin{aligned}
h_{*}^{G}\left(X \times E F^{\prime} \cup A \times E F, X \times E F^{\prime}\right) & =h_{*}^{G}\left(A \times E F, A \times E F^{\prime}\right) \quad \text { (by excision) } \\
& =h_{*}^{G}\left[F, F^{\prime}\right](A) .
\end{aligned}
$$

Let $F^{\prime \prime} \subset F^{\prime} \subset F$ be families in $G$ and consider the triple $(X \times E F, X \times$ $\left.E F^{\prime} \cup A \times E F, X \times E F^{\prime \prime} \cup A \times E F\right)$. Looking at the associated $h^{G}$ theory long exact sequence and using the fact that

$$
\begin{aligned}
h_{*}^{G}\left(X \times E F^{\prime} \cup A \times E F\right. & \left., X \times E F^{\prime \prime} \cup A \times E F\right) \\
& \cong h_{*}^{G}\left(X \times E F^{\prime}, X \times E F^{\prime \prime} \cup A \times E F^{\prime}\right) \quad \text { (by excision) } \\
& =h_{*}^{G}\left[F^{\prime}, F^{\prime \prime}\right](X, A)
\end{aligned}
$$

we obtain the following long exact sequence

$$
\begin{aligned}
\cdots \rightarrow h_{*}^{G}\left[F^{\prime}, F^{\prime \prime}\right](X, A) & \rightarrow h_{*}^{G}\left[F, F^{\prime \prime}\right](X, A) \rightarrow h_{*}^{G}\left[F, F^{\prime}\right](X, A) \\
& \rightarrow h_{*-1}^{G}\left[F^{\prime}, F^{\prime \prime}\right](X, A) \rightarrow \cdots
\end{aligned}
$$

Note. For bordism type theories we can also define $h_{*}^{G}\left[F, F^{\prime}\right](X, A)$ along the lines of Conner and Floyd [4] and Stong [13] -the resulting theory agrees with the one defined above, see the paper of tom Dieck [5].

If $F^{\prime}=\varnothing$ then we write $h_{*}^{G}\left[F, F^{\prime}\right](X, A)$ as $h_{*}^{G}[F](X, A)$, if furthermore $F=\{1\}$, the family consisting of just the trivial subgroup, then we write it as $h_{*}^{G}[$ free $](X, A)$.

If $F=$ All, the family consisting of all subgroups then $h_{*}^{G}[\mathrm{All}](X, A)=$ $h_{*}^{G}(X, A)$. 
6. Proof of main theorem. Recall that two families $F^{\prime} \subset F$ in $G$ are said to be adjacent if $F \backslash F^{\prime}$ only contains the conjugates of some single group, say $H$.

Throughout this section let $F^{\prime} \subset F$ be adjacent families in $G$ with $H \in$ $F \backslash F^{\prime}$. Returning to the commutative diagram $(\S 4)$, replacing $(X, A)$ by the pair $\left(E F \times X, E F \times A \cup E F^{\prime} \times X\right)$ gives the following commutative diagram.

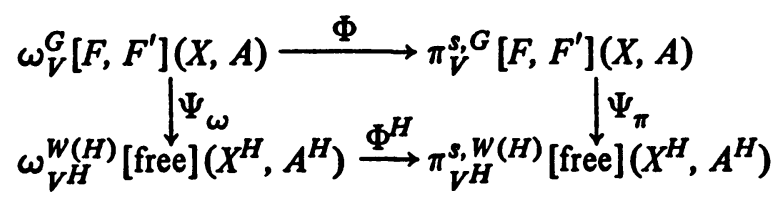

(Observe that $(E F \times X)^{H}=E W(H) \times X^{H}$ and $\left(E F \times A \cup E F^{\prime} \times X\right)^{H}=E W(H)$ $\times A^{H}$.)

THEOREM 6.1. $\Psi_{\omega}$ is an isomorphism.

Proof. We shall first define a map

$$
\Theta: \omega_{V^{H}}^{W(H)}[\text { free }]\left(X^{H}, A^{H}\right) \rightarrow \omega_{V}^{G}\left[F, F^{\prime}\right](X, A)
$$

Let $(N, t) \in \omega_{V H}^{W(H)}\left[\right.$ free] $\left(X^{H}, A^{H}\right)$, so $N$ is a $V^{H}$ framed $W(H)$ manifold and $t: N \rightarrow E W(H) \times X^{H}$ is a $W(H)$ equivariant map with $t(\partial N) \subset E W(H) \times A^{H}$. It follows that $N$ must be a free $W(H)$ manifold. Let $\left(V^{H}\right)^{\perp}$ denote the orthogonal complement of $V^{H}$ in $V$ and consider the following manifold

$$
Q=G \times \times_{N(H)}\left(N \times D\left(\left(V^{H}\right)^{\perp}\right)\right)
$$

which is easily seen to be a $V$ framed $G$ manifold. The isotropy subgroups in $Q$ are contained in the family $F$, hence there is a unique (up to $G$ homotopy) equivariant map $q_{1}: Q \rightarrow E F$. (The map $q_{1}^{H}: Q^{H} \rightarrow(E F)^{H}=E W(H)$ agrees with pt where $p: E W(H) \times X^{H} \rightarrow E W(H)$ is the projection map.)

We thus obtain a map

$$
q=q_{1} \times\left(G \times{ }_{N(H)} t\right): Q \rightarrow E F \times\left(G \times{ }_{N(H)} X^{H}\right) \hookrightarrow E F \times X .
$$

Since

$$
\partial Q=G \times \times_{N(H)}\left(\partial N \times D\left(\left(V^{H}\right)^{\perp}\right)\right) \cup G \times_{N(H)}\left(N \times S\left(\left(V^{H}\right)^{\perp}\right)\right)
$$

it follows that $q(\partial Q) \subset E F \times A \cup E F^{\prime} \times X$ and so $(Q, q)$ determines an element of $\omega_{V}^{G}\left[F, F^{\prime}\right](X, A)$. We define $\Theta$ by $\Theta(N, T)=(Q, q)$. Clearly $\Psi_{\omega} \Theta=$ id. The fact that $\Theta \Psi_{\omega}=$ id follows from the next two lemmas.

LEMMA 6.2. If $M$ is a $V$ framed $G$ manifold then the normal bundle of $M^{H}$ in $M$ is trivial and is given by $M^{H} \times\left(V^{H}\right)^{\perp}$. 
Proof. $T M \oplus\left(\mathrm{R}^{n} \times M\right) \cong(V \times M) \oplus\left(\mathrm{R}^{n} \times M\right)$ so

$$
T M^{H} \oplus\left(\mathbf{R}^{n} \times M^{H}\right) \cong\left(V^{H} \times M^{H}\right) \oplus\left(\mathbf{R}^{n} \times M^{H}\right)
$$

and

$$
\begin{aligned}
T M^{H} & \oplus \nu\left(M^{H}, M\right) \oplus\left(\mathrm{R}^{n} \times M^{H}\right) \cong\left(T M \oplus\left(\mathrm{R}^{n} \times M\right)\right) \mid M^{H} \\
& \cong\left((V \times M) \oplus\left(\mathrm{R}^{n} \times M\right)\right) \mid M^{H} \cong\left(V \times M^{H}\right) \oplus\left(\mathrm{R}^{n} \times M^{H}\right) \\
& \cong\left(V^{H} \times M^{H}\right) \oplus\left(\left(V^{H}\right)^{\perp} \times M^{H}\right) \oplus\left(\mathrm{R}^{n} \times M^{H}\right),
\end{aligned}
$$

which implies that $\nu\left(M^{H}, M\right)$ is $M^{H} \times\left(V^{H}\right)^{\perp}$ since the $H$ representation in $T M^{H}$ $\oplus\left(\mathrm{R}^{n} \times M^{H}\right)$ is $H$ trivial but not so in $\nu\left(M^{H}, M\right)$.

The next lemma is the analogue of Lemma 5.1 in [13].

LEMMA 6.3. Let $(M, f)$ and $\left(M^{\prime}, f^{\prime}\right)$ be elements of $\omega_{V}^{G}\left[F, F^{\prime}\right](X, A)$ and suppose that $M^{\prime}$ is a regularly embedded submanifold of $M$ with $f \mid M^{\prime}=f^{\prime}$. If every point of $M \backslash M^{\prime}$ has isotropy group belonging to $F^{\prime}$ then these elements represent the same class in $\omega_{V}^{G}\left[F, F^{\prime}\right](X, A)$.

Proof. Consider $M \times I$ where $I$ is the unit interval, with $r: M \times I \rightarrow$ $E F \times X$ given by $r(m, t)=f(m)$. We have

(i) $M \times I$ is a $V \oplus R$ framed $G$ manifold,

(ii) $M \cup\left(-M^{\prime}\right) \subset \partial(M \times I)$, with the induced $V$ trivializations on $\partial N$ restricted to $M,-M^{\prime}$ agreeing with that on $M,-M^{\prime}$ respectively,

(iii) $r: M \times I \rightarrow E F \times X$ is an equivariant map with

$$
\begin{aligned}
r\left(\partial(M \times I) \backslash\left(M \cup\left(-M^{\prime}\right)\right)\right) & =r\left((\partial M \times I \cup M \cup(-M)) \backslash\left(M \cup\left(-M^{\prime}\right)\right)\right) \\
& =r\left((\partial M \times I) \cup\left(-\left(M M^{\prime}\right)\right)\right) \subset E F \times A \cup E F^{\prime} \times X .
\end{aligned}
$$

THEOREM 6.4. $\Phi^{H}$ is an isomorphism.

Proof. This result follows from Lemma 2.1 and the fact that transversality works for $G$ maps between free $G$ spaces-see for example [8].

CoRollary 6.5. $\Phi$ is injective and $\Psi_{\pi}$ is surjective.

THEOREM 6.6. If $A=\varnothing$ then $\Psi_{\pi}$ is injective.

Proof. Suppose $f \in \pi_{V}^{s, G}\left[F, F^{\prime}\right](X)$ and that $\Psi_{\pi}(f)=0$, i.e. that $\Psi_{\pi}(f) \in \pi_{V}^{s, W}(H)[$ free $]\left(X^{H}\right)$ is $W(H)$ null homotopic.

Since $E F$ is the join of $G \times{ }_{N(H)} E W(H)$ with $E F^{\prime}$ we consider $G \times{ }_{N(H)}$ $E W(H)$ as being a subspace of $E F$. Also $X \times\left(G \times \times_{N(H)} E W(H)\right)$ is a subspace of $D(k W) \times X \times E F$ in the obvious way, and hence a subspace of $S^{k W} \wedge X \wedge$ $\left(E F / E F^{\prime}\right)$. 
Let $L=f^{-1}(X \times(G \times N(H) E W(H)))$ and let $*$ denote the base point of $S^{k W} \wedge X \wedge\left(E F / E F^{\prime}\right)$; then $L \cap f^{-1}(*)=\varnothing$. We shall show that $L=\varnothing$. Let $a$ be some point of $L$ and let $G_{a}$ denote the isotropy subgroup at $a$, then either

(I) $G_{a} \notin F$,

(II) $G_{a} \in F \backslash F^{\prime}$, or

(III) $G_{a} \in F^{\prime}$.

In case (I) $G_{a} \notin F$ then $G_{f(a)} \notin F$ since $G_{a} \subset G_{f(a)} \lessgtr H \in F$, and hence this case does not arise. In case (II) $G_{a} \in F \backslash F^{\prime}$ means that $G_{a}$ is conjugate to $H$ and $f(a) \in\left(S^{k W} \wedge X \wedge\left(E F / E F^{\prime}\right)\right)^{G_{a}}$ which by assumption on $\Psi_{\pi}(f)$ may be assumed to be the base point and hence $a \notin L$, so this case does not arise. Finally in case (III) $G_{a} \in F^{\prime}$, but $f$ restricted to such points factors through $\left(D(k W) \times X \times E F^{\prime}\right)$ which is in the base point of $\left(S^{k W} \wedge X \wedge\left(E F / E F^{\prime}\right)\right)$, thus this case also does not arise.

It follows that $L=\varnothing$, in other words $f^{-1}\left(X \times\left(G \times{ }_{N(H)} E W(H)\right)\right)=\varnothing$. Since the complement of $X \times\left(G \times{ }_{N(H)} E W(H)\right)$ in $S^{k W} \wedge X \wedge\left(E F / E F^{\prime}\right)$ is $G$ contractible it follows that $f$ is $G$ null homotopic.

Corollary 6.7. $\Phi$ is an isomorphism.

Proof. If $A=\varnothing$, then this follows immediately from Theorems 6.1, 6.4 and 6.6. In general the long exact sequence 5.1 and the five lemma provide a proof.

We have shown that $\Phi$ is an isomorphism for every pair of adjacent families. Since $G$ is a finite group we can find families $\varnothing=F_{1} \subset F_{2} \subset \cdots \subset F_{n}=$ All, such that $F_{i} \subset F_{i+1}$ are adjacent families in $G$. So by induction and the five lemma on the long exact sequence 5.2 we can show that $\Phi$ is an isomorphism for all pairs $F^{\prime} \subset F$ of families in $G$. In particular for $\varnothing \subset$ All. This completes the proof of the result that equivariant framed bordism is the same as equivariant stable homotopy.

\section{REFERENCES}

1. M. F. Atiyah, $K$-theory, Benjamin, New York, 1967. MR 36 \#1130.

2. G. E. Bredon, Introduction to compact transformation groups, Pure and Appl. Math., vol. 46, Academic Press, New York, 1972.

3. P. E. Conner and E. E. Floyd, Differentiable periodic maps, Ergebnisse der Mathematik und ihrer Grenzgebiete, N. F., Band 33, Academic Press, New York; Springer-Verlag, Berlin, 1964. MR 31 \#750. \#3587.

4. - Maps of odd period, Ann. of Math. (2) 84 (1966), 132-156. MR 34

5. T. tom Dieck, Orbittypen und äquivariante Homologie. I, Arch. Math. 23 (1972), 307-317. MR $46 \# 10017$.

6. H. Hauschild, Bordismentheorie stabil gerahmter G-Mannigfaltigkeiten, Math. Z. 139 (1974), 165-172.

7. C. Kosniowski, Equivariant cohomology and stable cohomotopy, Math. Ann. 210 (1974), 83-104. 
8. P. S. Landweber, On equivariant maps between spheres with involutions, Ann. of Math. (2) 89 (1969), 125-137. MR 38 \#6589.

9. J. J. O'Connor, D. Phil. Thesis, University of Oxford.

10. R. S. Palais, The classification of G-spaces, Mem. Amer. Math. Soc. No. 36 (1960). MR 31 \#1664.

11. R. L. Rubinsztein, On the equivariant homotopy of spheres, Institute of Math., Polish Academy of Sciences, Preprint no. 58, 1973.

12. G. Segal, Equivariant stable homotopy theory, Proc. Internat. Congress Math. (Nice, 1970), vol. 2, Gauthier-Villars, Paris, 1971, pp. 59-63.

13. R. E. Stong, Unoriented bordism and actions of finite groups, Mem. Amer. Math. Soc. No. 103 (1970). MR 42 \#8522.

SCHOOL OF MATHEMATICS, UNIVERSITY OF NEWCASTLE UPON TYNE, NEWCASTLE UPON TYNE, NEI TRU ENGLAND 